\title{
Putting a brake on stress signaling: miR-625-3p as a biomarker for choice of therapy in colorectal cancer
}

\begin{abstract}
"Ultimately, it should also be feasible to develop a simple predictive test for oxaliplatin response in colorectal cancer, which could guide the oncologist in selecting an optimal first-line treatment."
\end{abstract}

\begin{abstract}
First draft submitted: 27 September 2016; Accepted for publication: 27 September 2016; Published online: 25 October 2016
\end{abstract}

Keywords: biomarker $\bullet$ chemotherapeutics $\bullet$ colorectal cancer $\bullet$ miR-625-3p • oxaliplatin - resistance

Colorectal cancer (CRC) continues to be one of the leading cancer types worldwide [1]. When metastasized the disease is generally incurable and only treated with palliative intent. Metastatic CRC (mCRC) is primarily treated with 5-fluorouracil and leucovorin administrated together with either the topoisomerase inhibitor irinotecan (FOLFIRI) or with the alkylating cytostatic oxaliplatin (XELOX/FOLFOX). Although these treatment regimens have significantly improved both disease-free and overall survival, drug resistance is still a major problem, and consequently, only half of the treated mCRC patients obtain an objective response to the selected first-line treatment [2].

Multiple cellular resistance mechanisms in CRC have been described [3]. These include increased drug efflux or drug metabolism as well as alterations in the activity of repair pathways, or, in the case of targeted therapy, alterations in key proteins of the targeted pathway. Despite of this knowledge, the only predictive marker that is widely used in the clinic is the mutational status of the $K R A S$ gene; constitutive active mutations in this gene render drugs that target EGFR (an upstream activator of KRAS) ineffective [4].

In order to meet today's high expectations of personalized therapy there is thus a dire need to identify predictive markers for the main-stay drugs 5-fluorouracil, SN-38 (the active metabolite of irinotecan) and oxaliplatin.
We recently discovered that high expression of the miRNA miR-625-3p in tumors of $\mathrm{mCRC}$ patients is associated with more than six-times increased risk of not responding to oxaliplatin-based therapy [5]. miRNAs are a class of 20-22 nucleotides long, noncoding RNAs involved in regulating protein production by suppressing protein synthesis and by initiating mRNA degradation in a sequencespecific manner [6]. miRNAs are involved the control of numerous biological and physiological processes such as cell-cycle control, apoptosis as well as drug response [7].

Not much is known about the cellular functions of $m i R-625-3 p$. In a recent study by Zheng et al., it was found that $m i R-625-3 p$ increased the migration and invasion of CRC cell lines [8]. These functions were attributed to miR-625-3p-mediated downregulation of the SCAI protein encoded by SCAI (suppressor of cancer cell invasion).

To gain insight into miR-625-3p signaling and its association to oxaliplatin resistance, we have conducted mechanistic studies and molecular profiling in CRC cells with inducible expression of miR-625-3p [9]. This showed that miR-625-3p causally induces oxaliplatin resistance, and additionally, identified multiple drug response processes dysregulated as a consequence of miR-625-3p overexpression. The MAPK family was identified by phosphoproteome profiling as a key pathway class dysregulated by $m i R-625-3 p$. In addition, and likely
Epigenomics

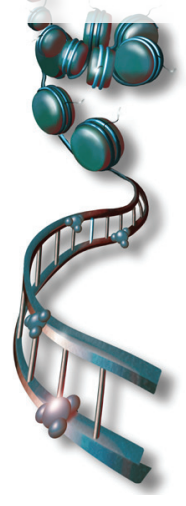

Iben Lyskjær

Department of Molecular Medicine, Department of Clinical Medicine, Aarhus University Hospital, Palle Juul-Jensens Boulevard 99 , DK-8200 Aarhus, Denmark

Mads H Rasmussen Department of Molecular Medicine, Department of Clinical Medicine, Aarhus University Hospital, Palle Juul-Jensens Boulevard 99, DK-8200 Aarhus, Denmark

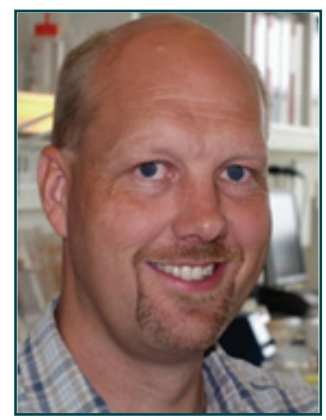

Claus L Andersen Author for correspondence: Department of Molecular Medicine, Department of Clinical Medicine, Aarhus University Hospital, Palle Juul-Jensens Boulevard 99, DK-8200 Aarhus, Denmark cla@clin.au.dk

$\begin{array}{ll}\text { Future } \because & \text { fSS } \\ \text { Medicine } \% \text { part of } & \end{array}$ 
as a consequence of aberrant MAPK signaling, cells overexpressing miR-625-3p appear to bypass downregulation of cytokine-dependent kinase activity upon oxaliplatin exposure, consistent with the observation that $m i R-625-3 p$ induces increased proliferation and survival in oxaliplatin-containing medium [9].

Explaining miR-625-3p-mediated dysregulation of MAPK signaling, MAP2K6 was found to be a direct and functional target of $m i R-625-3 p$. Consequently, increasing $m i R-625-3 p$ in CRC cells results in decreased MAP2K6 levels [9].

MAP2K6 (also known as MKK6), together with MAP2K3 (MKK3), is responsible for dual phosphorylation and activation of the p38 MAPK family of kinases. p38 $\alpha$ (MAPK14) is the most ubiquitously expressed family member, and its signaling is central for a bewildering range of biological functions including cytokine and growth factor signaling as well as for conveying cellular and environmental stress responses [10].

The role of p38 $\alpha$ in drug response has been intensively studied; although $\mathrm{p} 38 \alpha$ long has been recognized as a positive regulator of stress-induced apoptosis in transformed and nontransformed cells, studies have also shown its implication in mediating survival and resistance to drug treatment [10].

"This opens up for the possibility of administrating adjuvant anti-miR-625 treatment in combination with oxaliplatin to metastatic colorectal cancer patients with a high miR-625-3p level in order to potentiate the efficacy of therapy."

MAP2K6 is a substrate for ASK1 (also known as MAP3K5), a MAPK kinase kinase that becomes activated upon genotoxic stress [11]. This, in turn, leads to p38 $\alpha$ phosphorylation and - depending on context - possible induction of apoptosis via downstream effectors. When $\mathrm{p} 38 \alpha$ function is inhibited, the ability of platinum drugs such as oxaliplatin to induce apoptosis is diminished $[9,12,13]$. The oxaliplatin resistance observed in mir-625-3p-expressing CRC cells is in line with this, as $m i R-625-3 p$ expression leads to decreased levels of MAP2K6, the upstream activator of $\mathrm{p} 38 \alpha$ and consequently, failure to induce a proper p38 $\alpha$-dependent apoptotic signaling cascade [9].

During the last two decades, a surprising role for p38 $\alpha$ signaling has emerged that connects growth factor receptor signaling and production of reactive oxygen species (ROS) with the efficacy of combination therapy using EGFR inhibitors together with oxaliplatin.

As a consequence of genotoxic stress, for example, caused by oxaliplatin, p38 $\alpha$ induces apoptosis. This effect is augmented in cells with increasing levels of the $\mathrm{H}_{2} \mathrm{O}_{2}$ ROS species, possible via enforced
ASK1-MAP2K6 activation [14,15]. Hence, the cellular ROS level appears to be an important determinant for the cytotoxicity of genotoxic drugs [16,17]. Interestingly, external stimuli of growth receptors are associated with the production of low levels of ROS, an activity important for maintenance of normal cellular ROS homeostasis. Abnormal growth receptor pathway activity, such as from constitutive RAS proteins in cancer cells leads to aberrantly increased ROS levels, and can result in a synergistic effect of genotoxic drugs [18]. This interaction between ROS and p38 $\alpha$-induced apoptosis has been suggested to explain, at least partly, why some patients do not benefit from combination therapy involving oxaliplatin and cetuximab, the latter targeting EGFR, and consequently lowering ROS production $[13,18]$.

Remarkably, in contrast to the p38 $\alpha$-dependent cytotoxicity of oxaliplatin, SN-38 does not require ROS for induction of apoptosis [13]. On the contrary, p38 $\alpha$ activation is positively associated with resistance to SN-38 in vitro and in CRC patients [19]. Although the exact mechanism remains to be elucidated, p38 $\alpha$-induced survival autophagy is involved in resistance to $\mathrm{SN}-38$ [20], which perhaps explains the opposite effect of $\mathrm{p} 38 \alpha$ activation on response as compared with oxaliplatin. An intriguing question is whether CRC cells with reduced $\mathrm{p} 38 \alpha$ signaling as a consequence of $m i R-625-3 p$ upregulation are hypersensitive to $\mathrm{SN}-38$ treatment due to a diminished ability to induce survival autophagy? Further in vitro and in vivo studies would be needed in order to address this.

Several clinical perspectives of the relationship between miR-625-3p and MAP2K6-p38 $\alpha$ stress signalling can be envisioned. Exposure of resistant CRC cells ectopically expressing $m i R-625-3 p$ to an antagonist of $m i R-625-3 p$ (an anti-miR) reactivates oxaliplatin-induced apoptosis [9]. This opens up for the possibility of administrating adjuvant anti-miR-625 treatment in combination with oxaliplatin to mCRC patients with a high miR-625-3p level in order to potentiate the efficacy of therapy. An approach that may become reality in the near future as the clinical efficacy and safety of using anti-miRs to reduce the expression of specific disease-associated miRNAs have already been reported [21].

Ultimately, it should also be feasible to develop a simple predictive test for oxaliplatin response in $\mathrm{CRC}$, which could guide the oncologist in selecting an optimal first-line treatment. Such test might be based either on the level or activity of MAP2K6-p38 signaling, or on the expression level of miR-625-3p itself. In the latter case, a blood-based test may even be achievable since it is well known that miRNAs 
are relatively stable in the circulation compared with other biomolecules such as mRNA [22].

To pave the way for using miR-625-3p clinically, it would be necessary to conduct a prospective randomized trial comparing a random assignment to oxaliplatin or SN-38-based therapy against a treatment decision based on the miR-625-3p status (or MAP2K6-p38 $\alpha$ ) in individual patients. Approximately $20 \%$ of mCRC patients have elevated levels of miR-625-3p [5], and accordingly, these should be administrated an SN-38-based therapy. Such study would determine the clinical benefits, in terms of increased response rates and longer progression-free survival, of a $m i R-625-3 p / \mathrm{MAP} 2 \mathrm{~K} 6 / \mathrm{p} 38 \alpha$-guided treatment decision.

In conclusion, $m i R-625-3 p$ is a novel promising biomarker that hopefully may benefit CRC patients. By regulating MAP2K6, miR-625-3p impacts on the hinge of a central stress signaling pathway, which is part of the cellular response to drug treatment. A functional understanding of the regulation of

\section{References}

1 Siegel R, Desantis C, Jemal A. Colorectal cancer statistics, 2014. CA Cancer J. Clin. 64(2), 104-117 (2014).

2 Schmoll HJ, Van Cutsem E, Stein A et al. ESMO consensus guidelines for management of patients with colon and rectal cancer. a personalized approach to clinical decision making. Ann. Oncol. 23(10), 2479-2516 (2012).

3 Hammond WA, Swaika A, Mody K. Pharmacologic resistance in colorectal cancer: a review. Ther. Adv. Med. Oncol. 8(1), 57-84 (2016).

4 Misale S, Yaeger R, Hobor S et al. Emergence of KRAS mutations and acquired resistance to anti-EGFR therapy in colorectal cancer. Nature 486(7404), 532-536 (2012).

5 Rasmussen MH, Jensen NF, Tarpgaard LS et al. High expression of microRNA-625-3p is associated with poor response to first-line oxaliplatin based treatment of metastatic colorectal cancer. Mol. Oncol. 7(3), 637-646 (2013).

6 Bartel DP. MicroRNAs: genomics, biogenesis, mechanism, and function. Cell 116(2), 281-297 (2004).

7 Thomas J, Ohtsuka M, Pichler M, Ling H. MicroRNAs: clinical relevance in colorectal cancer. Int. J. Mol. Sci. 16(12), 28063-28076 (2015).

8 Zheng H, Ma R, Wang Q et al. MiR-625-3p promotes cell migration and invasion via inhibition of SCAI in colorectal carcinoma cells. Oncotarget 6(29), 27805-27815 (2015).

9 Rasmussen MH, Lyskjaer I, Jersie-Christensen RR et al. miR-625-3p regulates oxaliplatin resistance by targeting MAP2K6-p38 signalling in human colorectal adenocarcinoma cells. Nat. Commun. 7, 12436 (2016).

10 Cuadrado A, Nebreda AR. Mechanisms and functions of p38 MAPK signalling. Biochem. J. 429(3), 403-417 (2010). drug response pathways does not only enable us to develop biomarkers for today's therapy decisions, but should also facilitate the design of improved drugs for tomorrow's oncological challenges.

\section{Financial \& competing interests disclosure}

The authors have no relevant affiliations or financial involvement with any organization or entity with a financial interest in or financial conflict with the subject matter or materials discussed in the manuscript. This includes employment, consultancies, honoraria, stock ownership or options, expert testimony, grants or patents received or pending, or royalties.

No writing assistance was utilized in the production of this manuscript.

\section{Open access}

This work is licensed under the Creative Commons Attribution-NonCommercial 4.0 Unported License. To view a copy of this license, visit http://creativecommons.org/ licenses/by-nc-nd/4.0/

11 Chen Z, Seimiya H, Naito M et al. ASK1 mediates apoptotic cell death induced by genotoxic stress. Oncogene 18(1), 173-180 (1999).

12 Liu HF, Hu HC, Chao JI. Oxaliplatin down-regulates survivin by p38 MAP kinase and proteasome in human colon cancer cells. Chem. Biol. Interact. 188(3), 535-545 (2010).

13 Santoro V, Jia R, Thompson $\mathrm{H}$ et al. Role of reactive oxygen species in the abrogation of oxaliplatin activity by cetuximab in colorectal cancer. J. Natl. Cancer Inst. 108(6), djv394 (2016).

14 Tobiume K, Matsuzawa A, Takahashi T et al. ASK1 is required for sustained activations of JNK/p38 MAP kinases and apoptosis. EMBO Rep. 2(3), 222-228 (2001).

15 Ichijo $\mathrm{H}$, Nishida $\mathrm{E}$, Irie $\mathrm{K}$ et al. Induction of apoptosis by ASK1, a mammalian MAPKKK that activates SAPK/ JNK and p38 signaling pathways. Science 275(5296), 90-94 (1997).

16 Laurent A, Nicco C, Chereau C et al. Controlling tumor growth by modulating endogenous production of reactive oxygen species. Cancer Res. 65(3), 948-956 (2005).

17 Benhar M, Dalyot I, Engelberg D, Levitzki A. Enhanced ROS production in oncogenically transformed cells potentiates c-Jun N-terminal kinase and p38 mitogenactivated protein kinase activation and sensitization to genotoxic stress. Mol. Cell. Biol. 21(20), 6913-6926 (2001).

18 Dahan L, Sadok A, Formento JL, Seitz JF, Kovacic H. Modulation of cellular redox state underlies antagonism between oxaliplatin and cetuximab in human colorectal cancer cell lines. Br. J. Pharmacol. 158(2), 610-620 (2009).

19 Paillas S, Boissiere F, Bibeau F et al. Targeting the p38 MAPK pathway inhibits irinotecan resistance in colon adenocarcinoma. Cancer Res. 71(3), 1041-1049 (2011). 
20 Paillas S, Causse A, Marzi L et al. MAPK14/p38alpha confers irinotecan resistance to TP53-defective cells by inducing survival autophagy. Autophagy 8 (7), 1098-1112 (2012).

21 Van Der Ree MH, Van Der Meer AJ, De Bruijne J et al. Long-term safety and efficacy of microRNA-targeted therapy in chronic hepatitis C patients. Antiviral Res. 111 53-59 (2014).

22 Creemers EE, Tijsen AJ, Pinto YM. Circulating microRNAs: novel biomarkers and extracellular communicators in cardiovascular disease? Circ. Res. 110(3), 483-495 (2012). 